\title{
Study on the Timing of Degassing for Reproducible Preparation of Polymer-Based Monolithic Columns
}

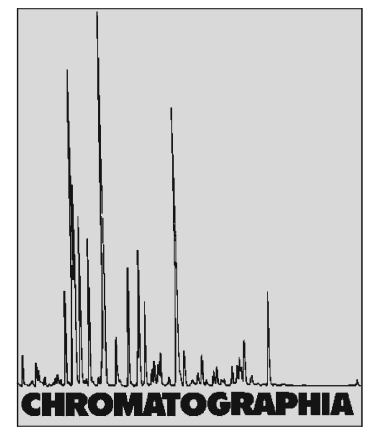

\author{
Kaname Ohyama ${ }^{1}$, Tomoyuki Sueyoshi ${ }^{1}$, Naoya Kishikawa ${ }^{1}$, Kenichiro Nakashima ${ }^{2}$, \\ Naotaka Kuroda, ${ }^{1, \infty}$ \\ 1 Department of Environmental and Pharmaceutical Sciences, Graduate School of Biomedical Sciences, Nagasaki University, \\ 1-14 Bunkyo-machi, Nagasaki 852-8521, Japan; E-Mail: n-kuro@nagasaki-u.ac.jp \\ 2 Department of Clinical Pharmacy, Graduate School of Biomedical Sciences, Nagasaki University, 1-14 Bunkyo-machi, \\ Nagasaki 852-8521, Japan
}

\begin{abstract}
The influence of timing of degassing during the preparation procedure on reproducible preparation of polymer-based monolithic columns was investigated. The degassing of each solvent before preparing the polymerization mixture exhibited good reproducibility and was found to be appropriate for the monolithic column without any change in the composition of the polymerization mixture.
\end{abstract}

\section{Keywords}

Capillary electrochromatography

Polymer-based monolithic column

\section{Introduction}

Organic polymer-based monolithic columns have been successfully used in LC and capillary electrochromatography (CEC) [1-6]. Polymer-based monoliths are attractive because they possess a wide variety of monomers and can be used within a broad pH-range where they are chemically stable. They are prepared by a single-step copolymerization with monomer, cross-linker, pore- forming solvent and initiator. However, unreproducible preparation of the monolithic columns is sometimes experienced, but there are only a few data on the reproducibility for the preparation of acrylate monolithic columns in the literature [7, 8]. Especially, batch-to-batch reproducibility is a matter of concern. The preparation procedure for polymer monolith, including preparation of polymerization mixture, filling of capillaries as well as the polymerization process, is very simple and the influencing factors to reproducibility are limited. Among these, a degassing is usually prepared for removing oxygen from reaction mixtures because oxygen acts as an inhibitor in free-radical polymerization [9]. In the preparation of polymer monolithic columns, the degassing is always carried out after mixing all the materials and the study on the timing of degassing during the preparation procedure has never been done.

In the current study, the influence of the timing of degassing (i.e. before or after mixing all the materials) on the preparation of polymer-based monolithic columns was investigated.

\section{Experimental}

\section{Chemicals}

Ethylene dimethacrylate (EDMA), 2-acrylamido-2-methyl-1-propanesulfonic acid (AMPS), $\alpha, \alpha^{\prime}$-azobisisobutyronitrile (AIBN), 1,4-butanediol and 3-(trimethoxysilyl)propyl methacrylate were obtained from Nacalai Tesque (Kyoto, Japan). 1-Adamantyl-( $\alpha$-trifluoromethyl) acrylate (MAF-ADE) was kindly gifted 
Table 1. Repeatability and reproducibility of preparation of ADM-functionalized monolithic columns expressed as RSD of EOF mobility, retention time and retention factor

\begin{tabular}{|c|c|c|c|c|c|}
\hline \multirow{3}{*}{$\begin{array}{l}\text { Parameter } \\
\text { Electroosmotic mobility }\end{array}$} & \multirow{3}{*}{$\begin{array}{l}\text { Repeatability (RSD) } \\
\text { Run-to-run }(n=7) \\
0.4\end{array}$} & \multicolumn{4}{|c|}{ Reproducibility (RSD) } \\
\hline & & \multicolumn{3}{|c|}{ Column-to-column $(n=5)$} & \multirow{2}{*}{$\begin{array}{l}\text { Batch-to-batch }(n=3) \\
3.5\end{array}$} \\
\hline & & $2.0^{\mathrm{a}}$ & $1.3^{\mathrm{b}}$ & $5.2^{\mathrm{c}}$ & \\
\hline $\begin{array}{l}\text { Benzene } \\
\text { (retention time) } \\
\text { (retention factor) }\end{array}$ & $\begin{array}{l}0.3 \\
2.6\end{array}$ & $\begin{array}{l}1.9^{\mathrm{a}} \\
5.7^{\mathrm{a}}\end{array}$ & $\begin{array}{r}2.3^{\mathrm{b}} \\
10.7^{\mathrm{b}}\end{array}$ & $\begin{array}{l}5.4^{\mathrm{c}} \\
7.1^{\mathrm{c}}\end{array}$ & $\begin{array}{l}3.3 \\
3.0\end{array}$ \\
\hline $\begin{array}{l}\text { Naphthalene } \\
\text { (retention time) } \\
\text { (retention factor) }\end{array}$ & $\begin{array}{l}0.4 \\
0.5\end{array}$ & $\begin{array}{l}2.1^{\mathrm{a}} \\
2.6^{\mathrm{a}}\end{array}$ & $\begin{array}{l}2.8^{\mathrm{b}} \\
4.1^{\mathrm{b}}\end{array}$ & $\begin{array}{l}5.8^{\mathrm{c}} \\
3.0^{\mathrm{c}}\end{array}$ & $\begin{array}{l}4.2 \\
3.8\end{array}$ \\
\hline $\begin{array}{l}\text { Anthracene } \\
\text { (retention time) } \\
\text { (retention factor) }\end{array}$ & $\begin{array}{l}0.6 \\
1.2\end{array}$ & $\begin{array}{l}1.9^{\mathrm{a}} \\
1.8^{\mathrm{a}}\end{array}$ & $\begin{array}{l}3.1^{\mathrm{b}} \\
3.2^{\mathrm{b}}\end{array}$ & $\begin{array}{l}6.2^{\mathrm{c}} \\
3.3^{\mathrm{c}}\end{array}$ & $\begin{array}{l}5.5 \\
6.1\end{array}$ \\
\hline
\end{tabular}

${ }^{\text {a }} \mathrm{N}_{2}$ degassing of each solvent before mixing all the materials

${ }^{\mathrm{b}} \mathrm{N}_{2}$ degassing of polymerization mixture

${ }^{\mathrm{c}}$ Ultrasonication degassing of polymerization mixture

from Tosoh Organic Chemical (Yamaguchi, Japan). 1-Propanol, acetic acid, sodium acetate, potassium dihydrogenphosphate, dipotassium hydrogenphosphate, benzene, naphthalene and anthracene were from Wako (Osaka, Japan). Thiourea was obtained from Kishida Chemical (Osaka). LC-grade acetonitrile (ACN) was purchased from Kanto Chemical (Tokyo, Japan).

\section{Electrochromatography}

All CEC experiments were performed on a CAPI-3200 system equipped with a photodiode array UV detector (Otsuka Electronics, Osaka). Fused-silica capillaries $(375 \mu \mathrm{m}$ o.d. $\times 75 \mu \mathrm{m}$ i.d. $)$ were obtained from Polymicro Technologies (Phoenix, AZ, USA). The mobile phase, prepared by mixing $5 \mathrm{mM}$ phosphate buffer (pH 7.0) and $\operatorname{ACN}(20 / 80, v / v)$, was degassed thoroughly prior to use. The separation voltage was set at $10 \mathrm{kV}$ and the injections were made by applying a voltage of $10 \mathrm{kV}$ for $8 \mathrm{~s}$.

\section{Column Preparation}

Monoliths were prepared by thermally initiated free-radical polymerization of the bulk monomer (MAF-ADE), a cross-linker (EDMA) and a monomer with a sulfonic acid group (AMPS) for the generation of electroosmotic flow (EOF). These monomers, pore-forming solvents (water, 1-propanol and 1,4butanediol) and AIBN were used following the recipe $[1,4,10]$, except for degassing timing. The degassing with nitrogen gas was performed as follows: flow rate, $0.1 \mathrm{~mL} \mathrm{~s}^{-1}$; nozzle diameter, $1.0 \mathrm{~mm}$; vessel, $15 \mathrm{~mL}$ glass vial. The gas inlet position was set at the bottom of the glass vial. The resulting columns were flushed first with water-ACN mixture $(20 / 80, v / v)$ to remove pore-forming solvents and unreacted monomers and then flushed with mobile phase by LC pump.

\section{Results and Discussion}

In all the reports dealing with polymer monolithic stationary phases, the degassing with nitrogen was always carried out after mixing the materials. However, in most cases, the polymerization mixture contains organic solvents as a poreforming solvent and thus, a part of the mixture can evaporate. This evaporation changes the composition of the polymerization mixture, resulting in unreproducible preparation of polymer monolith. When the total volume of polymerization mixture is lower, the risk is higher.

At first, the polymerization mixture $(1.5 \mathrm{~g})$ was weighed before and after the degassing to examine if the evaporation occurs with the degassing. As a result, a decrease of $15.5-21.3 \%$ in the total weight of polymerization mixture was observed. Furthermore, in order to identify which of the components evaporates, each pore-forming solvent (water, 1-propanol and 1,4-butanediol) was separately weighed before and after degassing, and water and 1-propanol were found to evaporate. These results suggest that the degassing should not be carried out on the polymerization mixture to avoid the composition change.

In this context, we proposed the degassing of each solvent for removing oxygen before mixing all materials. In order to verify this proposal, the repeatability and reproducibility of the monolithic columns were examined. Monolithic columns made from a polymerization mixture containing $11.8 \%$ MAF-ADE， $8.0 \% \quad$ EDMA, $\quad 0.4 \%$ AMPS, 8.0\% water, 47.0\% 1-propanol, $24.8 \%$ 1,4-butanediol were used. Each material in a solution, i.e. EDMA, water, 1-propanol and 1,4-butanediol, was bubbled with nitrogen for $20 \mathrm{~min}$. Then, the polymerization mixture was made by mixing all materials, which were followed by polymerization process within the capillaries.

The run-to-run repeatability of a monolithic column was evaluated by seven injections, while the column-tocolumn reproducibility was calculated 
from results obtained with five columns prepared from the same polymerization mixture. The reproducibility of monolithic columns $(n=3)$ prepared from three different polymerization mixtures with the same composition was also examined. The repeatability and reproducibility were expressed as relative standard deviations (RSD) of the chromatographic parameters, i.e. EOF mobility, retention time and retention factor, measured with the set of aromatic hydrocarbons (benzene, naphthalene and anthracene). As shown in Table 1, good reproducibilities were achieved for all tested parameters. RSD values comprised between 0.3 and $2.6 \%$ for run-torun repeatability and between 1.8 and $5.7 \%$ for column-to-column reproducibility were obtained in the electrochromatographic parameters. Satisfactory RSD values for the same parameters on batch-to-batch reproducibility $(<6.1 \%)$ were also achieved. For column-tocolumn reproducibility as shown in Table 1, the preparation procedure with degassing of each solvent exhibited better reproducibility than that with degassing after mixing all materials (RSD values $1.3 \sim 10.7 \%$ ) and that with degassing by ultrasonication (RSD values $3.0 \sim 7.1 \%$ ). Above all, the proposed procedure is appropriate for making the polymer monolith column without any change in the composition of the polymerization mixture.

\section{Conclusion}

The current study proposed the degassing of each solvent before making the polymerization mixture in order to prepare the monolithic column without any change in the composition and with high reproducibility. In this study, an adamantyl-functionalized monomer was utilized, but other reagents and polymerization conditions were the same as butyl methacrylate-based monolith which is the most widely used. Therefore, the knowledge obtained here can be transferred to the preparation of other polymer monoliths. The importance of degassing timing will be more pronounced when the total volume of polymerization mixture is lower on using expensive monomer materials.

\section{Acknowledgments}

K. O. is grateful for the support by the Special Coordination Funds for Promoting Science and Technology of the Ministry of Education, Culture, Sports, Science and Technology (MEXT). The authors thank Tosoh Organic Chemical for kindly supplying MAF-ADE monomer.

\section{References}

1. Peters EC, Petro M, Svec F, Fréchet JMJ (1998) Anal Chem 70:2288-2295. doi: $10.1021 /$ ac9713518

2. Švec F, Fréchet JMJ. In: Švec $F$, Tennikova TB, Deyl Z (eds) Monolithic materials. Elsevier, Amsterdam

3. Ferreira A, Bigan M, Blondeau (2003) React Funct Polym 56:123-136. doi: 10.1016/S1381-5148(03)00049-X

4. Eeltink S, Herrero-Martinez JM, Rozing GP, Schoenmakers PJ, Kok WTh (2005) Anal Chem 77:7342-7347. doi:10.1021/ ac051093b

5. Yan W, Gao R, Zhang Z, Yan C, Wang Q (2003) Chromatographia 57:819-823. doi:10.1007/BF02491771

6. Liu H, Row KH, Yang G (2005) Chromatographia 61:429-432. doi:10.1365/ s10337-005-0531-x

7. Grafnetter J, Coufal P, Tesařová E, Suchánková J, Bosáková Z, Ševčík J (2004) J Chromatogr A 1049:43-49. doi: 10.1016/j.chroma.2004.08.009

8. Cantó-Mirapeix A, Herrero-Martínez JM, Mongay-Fernández C, Simó-Alfonso EF (2008) Electrophoresis 29:3866-3874. doi:10.1002/elps.200800154

9. Zhang Z, Zhu X, Zhu J, Cheng Z, Zhu S (2006) J Polym Sci Part A Polym Chem 44:3343-3354. doi:10.1002/pola.21438

10. Ohyama K, Fukahori Y, Nakashima K, Sueyoshi T, Kishikawa N, Kuroda N (2010) J Chromatogr A 1217:1501-1505. doi:10.1016/j.chroma.2009.12.056 